\title{
EFEITOS DA DENSIDADE E PROPORÇÃO DE PLANTAS DE MILHO (Zea mays L.) E CARURU (Amaranthus retroflexus $\mathbf{L}$.) EM COMPETIÇÃO ${ }^{1}$
}

\author{
PEDRO J. CHRISTOFFOLETI ${ }^{2}$, RICARDO VICTORIA FILHO ${ }^{2}$
}

\begin{abstract}
RESUMO
A maioria dos estudos de competição, entre plantas daninhas e cultivadas, conduzidos nos últimos anos, procuram quantific ar a interferência que as plantas daninhas causam sobre as culturas; no entanto, poucos destes trabalhos estudam mecanistic amente os efeitos da densidade e da proporção de plantas em uma mistura de espécies, a importância da competição intra-e interespecífica e a diferenciação de nicho ecológico. Desta forma, foi desenvolvida a presente pesquisa com o objetivo principal de descrever as interações competitivas e os índices de competitividade en tre plantas de milho (Zea mays L.) e caruru (Amaranthus retroflexus $\mathrm{L}$ ). A metodologia utilizada foi a de um experimento substitutivo com densidade total de 400 plantas $/ \mathrm{m}^{2}$ e 5 proporções, além da

convencional de análise de experimentos substitutivos e pelo método da produção recíproca total e por planta. O milho foi um competidor muito mais agressivo que o caruru, sendo que para a planta cultivada a competição intraespecífica é mais importante que a competição intere specífica. O contrário é verdadeiro para o caruru, ou seja, a competição interespecífica é mais importante que a intraespecífica. Ambas espécies de plantas estão competindo pelos mesmos fatores de crescimento, pois o índice que mede a diferenciação de nicho ecológ ico é menor que 1,0 . A de terminação da influência da densidade e proporção de espécies em estudos de competição entre plantas é muito importante para a compreensão das interações competitivas.
\end{abstract} monocultura que variou de 50 a $800 \mathrm{plantas} / \mathrm{m}^{2}$, sendo conduzido no delineamento experimental de blocos casualizados, com quatro repetições. Os resultados obtidos foram analisados pelo método
Palavras chave: Experimento substitutivo, índices de competição, competição intraespecífica, competição interespecífica, nicho ecológico.

\section{ABSTRACT \\ Density and porportion effects among corn (Zea mays L.) and pigw eed (Amaranthus retroflexus $\mathbf{L}$.) under competition.}

Most of the competition studies between weeds and crops conducted lately quantify the interference that the weeds cause to crops; however, the se researches do not show mechanistically neither the effects of density and proportion of plants in a mixture of species, nor the importance of in tra and inter-specific competition and niche diferentiation. Therefore, this research was developed aiming to describe the competitive interaction and competitive indexes between corn (Zea mays L.) and pigweed (Amaranthus retroflexus L.) plants. The methodology was a replacement series experiment with a total density of $400 \mathrm{plants} / \mathrm{m}^{2}$ and 5 proportions, be sides the monoculture that varied from 50 to 800 plants $/ \mathrm{m} 2$, conducted in a randomized completely block design, four replications. The results were analyzed through conventional replacement series analysis,

1 Recebido para publicação em 14/11/95 e na forma revisada em 27/06/96.

2 Professores Dout or e Titular respectivamente do Departamento de Horticultura da Escola Superior de Agricultura "Luiz de Queiroz", Universidade de São Paulo, Av. Pádua Dias. 11, Caixa Postal 09, CEP 13.418-900, Piracicaba, SP. 
using reciprocal total and per plant yield. Corn was the superior competitor and the intra-specific competition was more important than the interspecific for this species. The reverse was true for pigweed. Both species were competing for the same natural resources since the niche diferentiation index was lower than 1,0 . The

\section{INTRODUÇÃO}

A determinação das interações competitivas entre espécies de plantas requer delineamentos experimentais e métodos de análise apropriados (Roush et al. 1989). Estas interações competitivas são af etadas por diversos fatores. Pitelli (1985) relata um modelo esquemático destes fatores, através de urna adaptação do modelo de Ble as da le (1960). Dentre os fatores ligados à comunidade infestante, a densidade de plantas é sem dúvida um dos fatores mais importantes, de tal forma que quanto maior for a densidade da comunidade infestante, maior será a quantidade de indivíduos que disputam os mesmos recursos do meio e, portanto, mais intensa será a competição sofrida pela cultura.

Nas áreas de produção agrícola, a densidade das plantas cultivadas é mantida constante, ao passo que a densidade das plantas daninhas varia de acordo com o nível de infestação encontrado no local; desta forma, obtém-se logicamente uma variação da proporção entre as espécies daninhas e da cultura. Assim, é importante, nos estudos de competição, não apenas medir a influência da densidade no processo competitivo, mas também a importáncia da variação na proporção entre as espécies.

Existem diversas metodologias usadas para estudar a competição entre plantas (Roush et al. 1989); no entanto, segundo levantamento feito por Zimdahl (1980) em mais de 600 trabalhos científicos publicados sobre competição de plantas daninhas com plantas cultivadas, todos eles procuram apenas quantificar a interferência que as plantas daninhas provocam nas culturas sem se preocupar com a compreens ão do processo de competição. No Brasil, a maioria das pesquisas de competição entre plantas daninhas e plantas influence of density and proportion of species in a competition study is very important in the understanding of competitive interactions.

Key words: Replacement series, competition index, intra-specific competition, interspecific competition, ecological niche.

cultivadas determinam o período crítico de competição (Christoffoleti, 1988). Desta forma é importante o uso de delineamentos experimentais e metodologias de análise que procurem entender o processo competitivo de forma mais mecanística, e não apenas quantificar as perdas.

Dentre as metodologias existentes, os experimentos substitutivos são uma alternativa para a compreensão do processo competitivo entre plantas, especialmente quando relacionado com o estudo do efe ito da densidade e da proporção entre plantas em uma comunidade infestante. Experimentos substitutivos são delineados de tal maneira que ex is te um controle da densidade e proporção das espécies em estudo (Harper, 1977 e Wit, 1960), mantendo constante a densidade e variando as proporções das duas espécies. Nestes tipos de experimentos as sume-se que a densidade total de plantas é suficientemente grande de tal forma que satisfaça a chamada "lei da produção final constante", onde a produção de biomassa por unidade de área é independente da densidade das plantas naquela área.

A interpretação dos dados de um experimento substitutivo resulta na medida da competitividade das espécies baseada na resposta relativa da produção de biomassa pela variação da proporção (Harper, 1977; McGilchrist \& Trenbath, 1974 e Wit \& Van Den Bergh, 1965). É possível calcular equações mate máticas e representações gráficas onde se estabelecem índices de competição intra-e interespecífica e diferenciação de nicho ecológico (Spitters, 1983 e Joliffe et al. 1984).

Desta forma, o objetivo desta pesquisa foi de quantificar a competitividade relativa da cultura do milho e da planta daninha caruru, através da medida do efeito da densidade e da proporção, usando experimento substitutivo.

Planta Daninha, v. 14, n. 1, 1996. 


\section{MATERIAL E MÉTODOS}

O experimento foi conduzido em casa-deve ge tação do De partamento de Horticultura da Escola Superior de Agricultura "Luiz de Queiroz", da Universidade de São Paulo, localizada no município de Piracicaba-SP, durante os meses de sete mbro a dezembro de 1994. Inicialmente foi instalado um experimento com as monoculturas de milho e caruru, ou seja tratamentos contendo apenas cada uma das espécies. Tanto o milho quanto o caruru foram semeados em caixas de $25 \mathrm{x}$ $15 \times 15 \mathrm{~cm}$, contendo substrato especialmente preparado com terra, areia e terriço. O número de plantas por caixa foi correspondente às densidades de 50,100, 200, 400 e 800 plantas $/ \mathrm{m}^{2}$ de cada uma das espécies estudadas, semeadas de maneira uniforme, ou seja eqüidistante. Foram feitas quatro repetições, conduzidas em um delineamento experimental de blocos ao acaso.

O experimento substitutivo foi instalado após colheita do experimento com as monoculturas, também conduzido em casa-de-ve ge tação, em caixas de $25 \times 15 \times 15 \mathrm{~cm}$, contendo uma densidade constante, porém com tratamentos representando diferentes proporções entre plantas de milho e caruru. As proporções foram 0:400; 100:300; 200:200, 100:300 e 400:0 representando respectivamente as quantidades de plantas de milho e caruru por metro quadrado.

As plantas encontravam-se também uniformemente distribuídas nas parcelas. Os tratamentos das proporções foram repetidos quatro vezes, atra vés de um delineamento de blocos ao acaso.

Em todos os experimentos o solo foi constituído de uma mistura em partes iguais de solo, areia e terriço retirado de áreas reflorestadas, com $\mathrm{pH}=5,5$. Durante a condução do ensaio foi fornecida água em quantidades suficientes para o bo m desenvolvimento tanto da planta daninha quanto da planta cultivada. As plantas foram então colhidas quando apresentavam 50 dias de idade. A biomassa da parte aérea das plantas foi coletada, depois seca em estufa de circulação renovada de ar a $60^{\circ} \mathrm{C}$ por dois dias, e pesada.
O experimento na forma de monocultura, foi analisado de acordo com Joliffe et al. (1984). Foi executada uma análise através de uma regressão linear do recíproco da produção por $\mathrm{m}^{2}$ como variável dependente (1/Y) e o recíproco da densidade como variável independente (1/D). A partir das equações matemáticas foram elaborados gráficos da regressão linear e da produção de biomassa $/ \mathrm{m}^{2}$ em função da densidade.

Os dados provenientes do experimento substitutivo foram inicialmente submetidos à anál is e convencional. Neste ti po de análise, os resultados são interpretados visualmente, através da análise de um gráfico contendo a resposta da produção relativa em relação à proporção (Harper, 1977; Wit, 1960 e Wit \& Van Den Bergh, 1965). As produções relativas para cada espécie foram calculadas pela produção a cada densidade e proporção, dividida pela produção média da monocultura naquela densidade.

Outro tipo de análise dos resultados do experimento substitutivo foi a metodologia desenvolvida por Spitters (1983), usando a produção média/planta obtida no experimento substitutivo (W=Y/D). Os dados proveniente de cada espécie foram então submetidos a uma análise de regressão linear, na forma das equações 1 e 2 .

$$
\begin{gathered}
\text { milho } \frac{l}{W_{m}}=A_{m}+B_{m m} \times D_{m}+B_{m c} \times D_{c} \\
\text { caruru } \frac{l}{W_{c}}=A_{c}+B_{c c} \times D_{c}+B_{c m} \times D_{m}
\end{gathered}
$$

Os coeficiente de competição intra$\left(\begin{array}{lllll}B_{m m} & \text { e } & B_{c c}\end{array}\right)$ e interespecífica $\left(\begin{array}{lll}B_{c m} & \text { e } & B_{m e}\end{array}\right)$ for am en tão utilizados para calcular a competitividade relativa das duas es pécies em es tu do (C), e o índice de diferenciação de nicho ecológico (IDN).

$$
\begin{gathered}
C_{m}=\frac{B_{m m}}{B_{m c}} ; C_{c}=\frac{B_{c c}}{B_{c m}} \\
I D N=\frac{\left(C_{m t}\right)}{\left(C_{c}\right)}
\end{gathered}
$$




\section{RESULTADOS E DISCUSSÃO}

Os resultados do experimento com a monocultura, analisados de acordo com a produção final constante, encontram-se representados nas Figuras 1 e 2. A produção final máxima (constante - Ymax) foi de $233 \mathrm{~g} / \mathrm{m}^{2}$ para o milho e de $222 \mathrm{~g} / \mathrm{m}^{2}$ para o caruru. As densidades que proporcionaram uma produçao de $50 \%$ da produção final constante $\left(\mathrm{K}_{\mathrm{n}}\right)$ foi de $79 \mathrm{plantas} / \mathrm{m}^{2}$ para o milho e de 144 plantas $/ \mathrm{m}^{2}$ para o caruru. Como a densidade correspondente ao $\mathrm{K}_{\mathrm{n}}$ do milho é bem menor em comparação com o $\mathrm{K}_{\mathrm{n}}$ do caruru, é evidente que o milho é mais sen sível à competição intraespecífica que o caruru. Através destes resultados observa -se que na densidade de $400 \mathrm{plantas} / \mathrm{m}^{2}$, tanto para o milho quanto para o caruru, a produção é se melhan te à produção máxima $\left(\mathrm{Y}_{\max }\right)$, conforme pode ser observado na Figura 2. Desta forma, o experimento substitutivo foi instalado com a densidade total de $400 \mathrm{plantas} / \mathrm{m}^{2}$, conforme exigência essencial deste tipo de delineamento experimental (Joliffe, el al. 1984).

$$
\text { Analisando os resultados contidos na }
$$

Figura 3, observa-se que os valores da produção obtidos na mistura das duas espécies desviaram

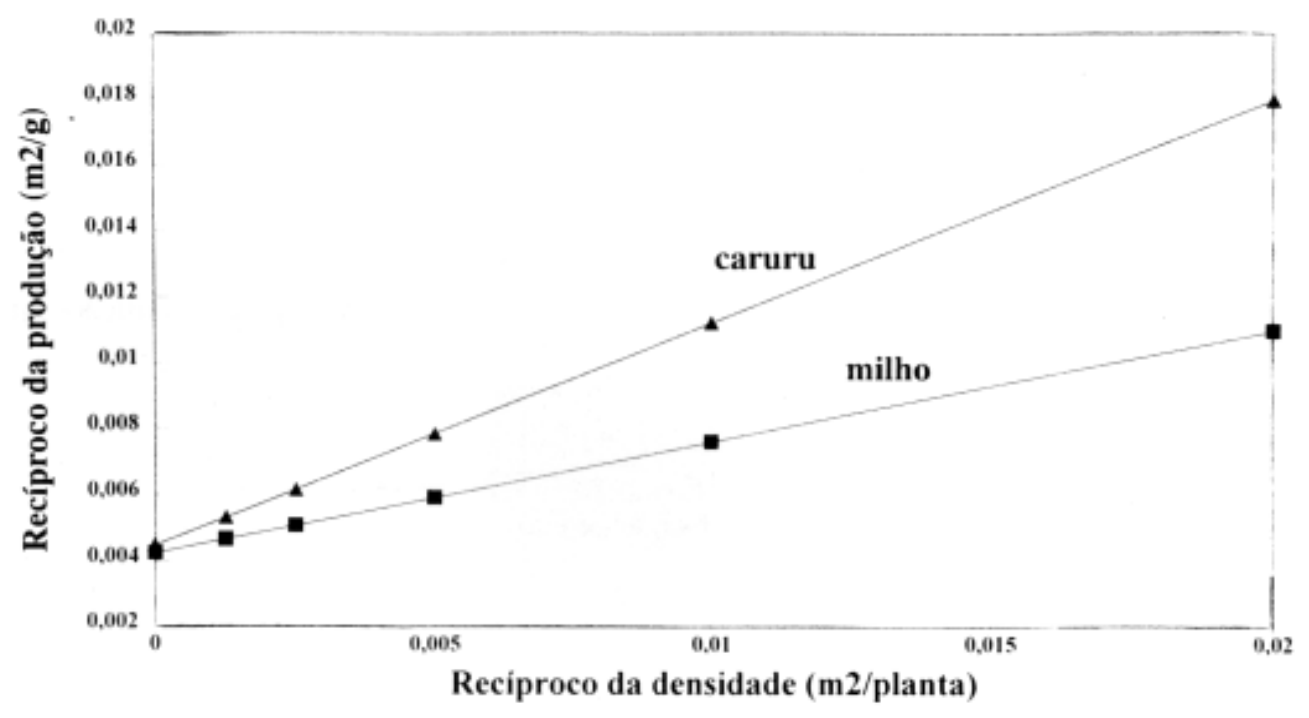

milho $-1 / \mathrm{Y}_{\mathrm{m}}=0.004254+0.3373^{*}\left(1 / \mathrm{D}_{\mathrm{m}}\right) \cdot \mathrm{R}^{2}=0.87$
caruru $-1 / \mathrm{Y}_{\mathrm{c}}=0.004493+0.647890^{*}\left(1 / \mathrm{D}_{\mathrm{c}}\right) \cdot \mathrm{R}^{2}=0.93$

FIGURA 1. Respostas da monocultura de milho e caruru representada pelo recíproco da produção em relação ao recíproco da densidade.

significativa mente da linha de produção esperada, ou seja a produção se não houvesse interação competitiva en tre as es pécies em competição. O milho produziu urna quantidade de biomassa acima da esperada, sendo que, o caruru produziu abaixo do esperado. Assim, o milho se beneficia da presença do caruru, pois a cultura é um competidor mais agressivo que a planta daninha. $\mathrm{O}$ milho prefere em sua vizinhança uma planta de caruru em relação a outra planta de milho.

A análise da produção recíproca dos resultados de biomassa por planta nos tratamentos do experimento substitutivo, conforme metodologia de senvol vida por Spit ters (1983), resultou nas equações 5 e 6 , a seguir: 


$$
\begin{aligned}
& \text { milho } \frac{l}{Y_{m}}=0,15767+0,00339 x D_{m}+0,00180 x D_{c} \rightarrow\left(R^{2}=0,95\right) \\
& \text { caruru } \frac{l}{Y_{c}}=0,64038+0,00367 x D_{c}+0,00959 x D_{m} \rightarrow\left(R^{2}=0,94\right)
\end{aligned}
$$

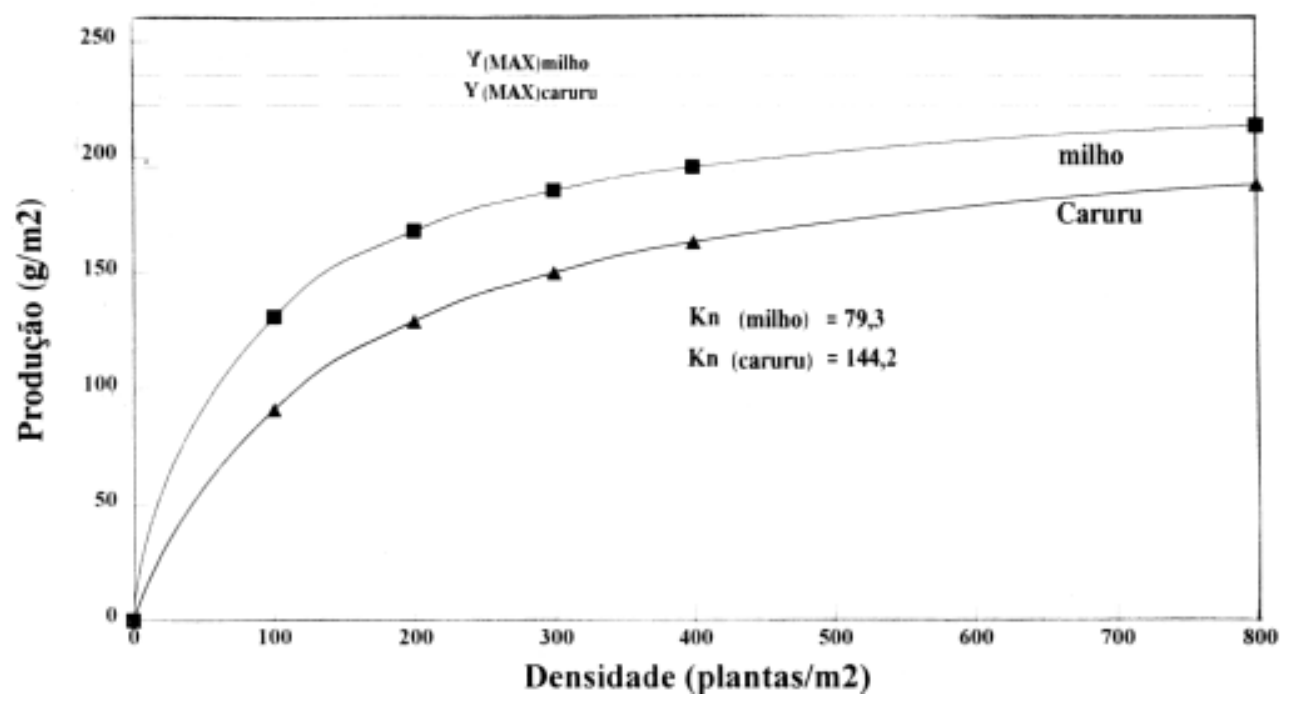

FIGURA 2. Respostas da monocultura de milho e caruru representada pela produção em relação à densidade.

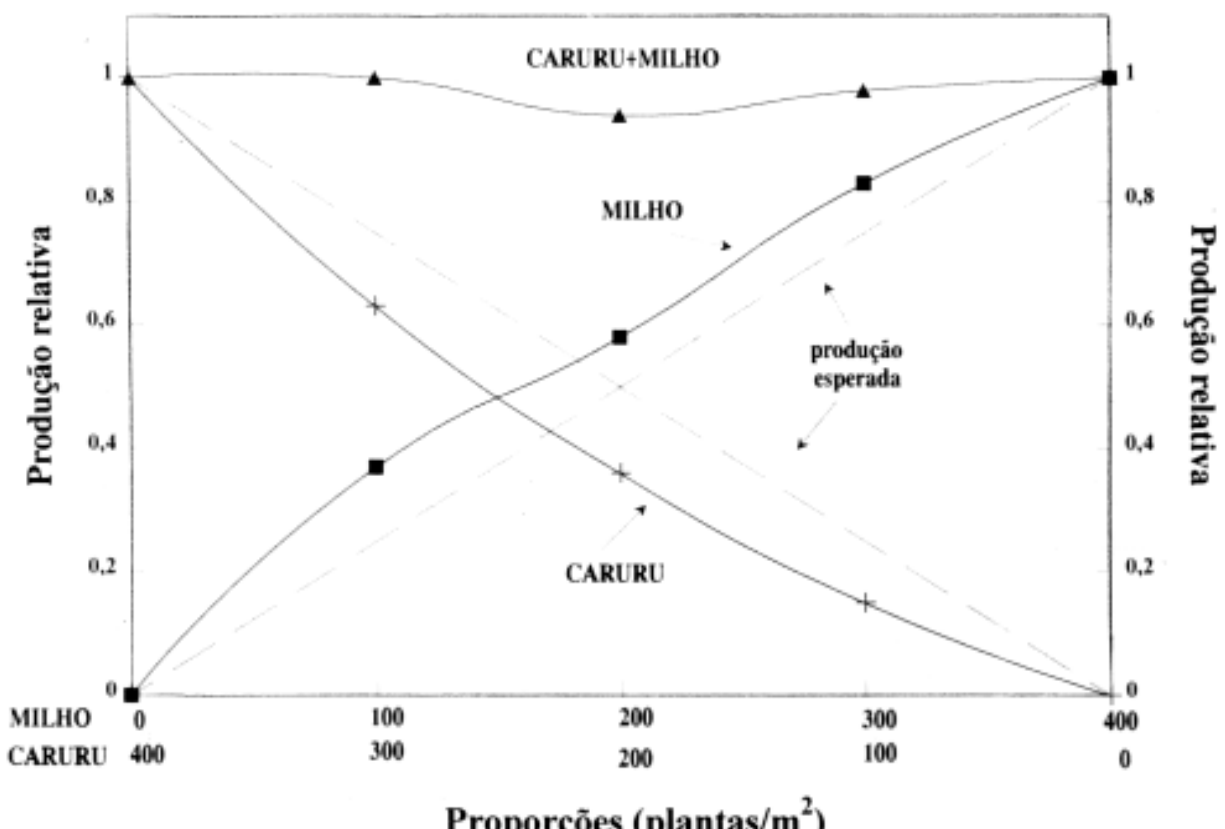

FIGURA 3. Diagrama da resposta da produção relativa média de milho e caruru em função da variação da proporção entre as duas espécies. As linhas tracejadas representam a hipótese da não interação. 
Os coeficientes para competição intra- e interespecífica dessas equações foram usados para calcular a competitividade relativa do milho e do caru ru (equação 3): $\mathrm{C}_{\mathrm{m}}=1,88 ; \mathrm{C}_{\mathrm{c}}=0,48$. Estes índices indicam que uma planta de milho equivale a 1,88 plantas de caruru ou que 0,48 plantas de milho corresponde a uma planta de caruru. Assim, o milho é melhor competidor que o caruru nas condições em que foi desenvolvido o presente experimento.

À partir dos índices de competitividade relativa pode ser calculado o índice de diferenciação de nicho ecológico (equação 4). Para o caso deste experimento o IDN $=0,71$. De acordo com Spitters (1983), quando o IDN > 1, 0 , is to sugere que ocorreu uma diferenciação de nicho ecológico para as duas espécies em convivência, de tal forma que as espécies produzem mais quando em mistura que comparad a com as monoculturas destas. Para o resultado deste experimento, o IDN $=0,71$, sugere que não houve nenhuma diferenciação de nicho ecológico entre o milho e o caruru e as duas espécies estão competindo pelos fatores ambientais de crescimento que se encontram em quantidades limitantes para o desenvolvimento de ambas.

\section{LITERATURA CITADA}

CHRISTOFFOLETI, P.J. Controle de Brachiaria decumbens Stapf e de Cyperus rolundos em área de cana-de-açúcar (Saccharum sp.) através da técnica de rotação com amendoim (A rachis hvpogaea) intergrada ao uso de herbicidas. Piracicaba: ES ALQ/USP, 1988, 117p. Dissertação de mestrado. 1988.
HARPER, J.L. Mixtures of species. I. Space and proportions. In: HARPER, J.L. The population biology of plants. Academic Press. London. 1977, p.237-304.

JOLIFFE, P.A., NINJAS, A.N., RUNECKLES, V.C. A reinterpretation of yield relationships in replacement series experiments. J. Appl. Ecol. v.21, p.227-243, 1984.

McGILCHRIST, C.A., TRENBATH, B.R. A revised analysis of plant competition experiments. Biometrics. v. 27, p.659-671, 1974.

PITELLI, R.A. Interferência de plantas daninhas em culturas agrícolas. Inf. Ag ropec., v.11, n.129, p.16-27, 1985.

ROUSH, M.L., RAD OSEVICH, S., WAGNER, R.G., MAXWELL, B., PETERSON, T.D. A comparision of methods for measuring effects of density and proportion in plant competition experiments. Weed Sci., v.37, n.2, p.268-275, 1989.

SPITTERS, C.J.T. An alternative approach to analysis of mixed cropping experiments. I. Estimation of competition effects. Neth. J. Agric. Sci. v.31, p.1-11, 1983.

ZIMD AHL, R.L. Weed and Crop Competition a review. International Plant Protection Center. 1980, 197p.

WIT, C.T. On competition. Versl. Land bouwk. D. Onderz. v.66, n.8, p.1-82, 1960.

WIT, C.T., VAN DEN BERGH, J.P.. Competition between herbage plants. Neth. J. Agric. Sci. v. 13, p.212-221, 1965. 